\title{
Coexistence and Market Tipping in a Diffusion Model of Open Source vs. Proprietary Software*
}

\author{
Riccardo Leoncini ${ }^{\dagger}$ \\ Francesco Rentocchini ${ }^{\ddagger}$ \\ Giuseppe Vittucci Marzetti ${ }^{\S}$
}

November 4, 2010

\begin{abstract}
A large number of studies has been canvassed by the growing rates of diffusion of Open Source Software. However, a formal analysis of the process of competition between open-source and proprietary software is still missing. We propose an epidemic model of innovation diffusion to deal with the different factors (profits for proprietary software and developers' motivations for open-source software) upon which such a process of competition ultimately depends Moreover, we add network effects and switching costs, together with the endogenisation of the parameters of the speed of diffusion influencing the final outcome. We show the conditions for an asymptotically stable equilibrium to exist, where both softwares coexist. When the propagation coefficient is endogenous, winner-take-all solutions are also likely. Furthermore, an increase in the level of the switching costs for one software increases the number of its adopters, while reducing that of the other one. If the negative network effects increase for one of the two softwares, then the equilibrium level of users of that software decreases.
\end{abstract}

Keywords: Increasing returns; Model of diffusion; Open-source software; Technological competition.

JEL Classification: L17; L86; O33.

\footnotetext{
*Previous versions of the paper were presented to the PRIN workshop on "Fragmentation and local development: interpretative models and policy implications", University of Padua, 3-4 June 2008, and to the EAEPE Conference, Rome, 6-8 November 2008. The authors gratefully acknowledge comments and suggestions from the participants, and in particular Giorgio Negroni, Marika Macchi and Ulrich Witt. The usual caveat applies. Francesco Rentocchini and Giuseppe Vittucci Marzetti gratefully acknowledge the financial support from the Autonomous Province of Trento (Grants 2006: TRACKs and FIC).

${ }^{\dagger}$ Dept. of Economics, University of Bologna, riccardo.leoncini@unibo.it

${ }^{\ddagger}$ Dept. of Economics, University of Trento, francesco.rentocchini@economia.unitn.it

$\S$ Dept. of Economics, University of Trento, giuseppe.vittucci@economia.unitn.it
} 


\section{Introduction}

The growth of adoption of Open Source Software (OSS) in recent years has attracted the attention of many scholars from different fields. A large number of case studies has been carried out to explain and empirically ground such a phenomenon. Among the others, topics such as the organization and ethos of the community of developers, their motivation to provide code for free and the birth of hybrid business models have been extensively examined by different branches of literature.

However, an issue not sufficiently investigated so far is related to how to model the process of OSS diffusion and its competition with Proprietary Software (PS). To our knowledge, only few contributions have tried to address such a crucial topic (e.g. Bonaccorsi and Rossi, 2003; Dalle and Jullien, 2003), though they rely on quite ad hoc models, rather than on more consolidated models of diffusion.

Our starting point is thus to model the process of competition between OSS and PS by means of an epidemic model, accounting for both demand and supply factors. Moreover, OSS fundamentals depend on a set of factors different from the PS ones (Lerner and Tirole, 2002), that is, reputational and communitarian factors (intrinsic and extrinsic motivations) versus profit motivated factors for PS. ${ }^{1}$

We use an epidemic model to depict the process of diffusion of software packages, which is affected by the size of the installed base. ${ }^{2}$ Indeed, it is quite straightforward for this class of models to depict within-technology positive network effects as a result of contagion (due, for instance, to word-ofmouth interactions). ${ }^{3}$ Furthermore, epidemic models are better suited than game theory models to describe situations in which, on the one side, there are important problems related to the effects of the transmission of knowledge among agents about the unknown characteristics of a technology, and, on the other side, the interest in strategic behaviour is almost non-existent, since OSS developers cannot "by definition" take into account how the evolution of their software will impact upon the competing one. Hence, it is easier to deal with the strategic behaviour of only one side (PS) considering that

\footnotetext{
${ }^{1}$ Nonetheless, a considerable number of large firms decided to enter into the software market in order to benefit from this process. Among the others, IBM, Novell and Dell are worth mentioning. This fact has led to the creation of a new type of hybrid business model characterised by the presence of for-profit companies benefiting from OSS solutions, mainly developed by the not-for-profit OSS communities they support (West, 2003; Fosfuri et al., 2008). Furthermore, an increasing number of countries all over the world has started discussing about the role OSS should have in public administration.

${ }^{2} \mathrm{An}$ increasing number of studies provides empirical evidence on the importance of network effects, both direct and indirect, in different market segments of the software industry. See, for instance, Gandal (1994) and Brynjolfsson et al. (1996) for spreadsheets; Chiaravutthi (2006) for browsers; and Gandal (1995) for PC software.

${ }^{3}$ As it will be clear in what follows, for the across-technology network effects we will introduce interoperability issues between the two technologies.
} 
some of its possible choices are unrelated to the competitor's reaction. At the same time, both PS and OSS diffusion are influenced by the presence of network effects, the level of interoperability, and the presence of switching costs.

Finally, if compared to standard diffusion models, our model endogenises the parameter influencing the speed of diffusion across the population of adopters. In so doing, from a theoretical perspective, our model offers a formal general treatment to solve more complex and more realistic diffusion models: it offers a way to properly deal with diffusion patterns whose speed can vary, as the process unfolds, since the interactions among the technologies contribute to change the incentives for consumers, as they progressively discover their characteristics.

The paper is structured as follows. In Section 2, we present the theoretical background: in particular, about the competition between PS and OSS, and about the models of diffusion (Section 2). We then discuss the general framework within which the two technologies compete. In particular, a set of three main features are considered: network effects, interoperability and switching costs. These characteristics are incorporated in the formal model developed in Section 3. The results are then presented in the following sections, where several variants of the model are discussed: (i) a base version characterised by constant propagation coefficients for the two technologies (Section 4); (ii) an extended version with changing propagation coefficients without network effects (Section 5.1) and then with network effects (Section 5.2). Section 6 concludes.

\section{Theoretical background}

Open source software has gained momentum thanks to several relevant "success stories", such as the Internet browser Firefox, the web server Apache, the suite OpenOffice and the mail sending protocol Fetchmail, that can be explained by several stylised facts (Leoncini, 2004). As a matter of fact, recent years have witnessed an increasing competition between proprietary and open source products. In particular, market shares of dominant proprietary vendors have experienced increasing pressure from OSS.

Although contributions on this topic are flourishing (see Rossi (2006) and Wheeler (2005) for thorough surveys), the literature dealing with the processes of competition and diffusion of two competing technologies (i.e. OSS vs PS) has attracted less effort, in part because of the difficulty to properly model it.

The contributions on this topic can be grouped into two main subsets. First, the majority of contributions are based on static models of industrial organization. In particular, several contributions stressed the importance of consumers in OSS production (Lakhani and von Hippel, 2003; Kuan, 2001), 
and model the competition between the two modes of production assuming that agents must decide between buying software and producing it, while other models (Johnson, 2002) refer to the decision of individual userprogrammers to contribute to software program as a decision to contribute to a public good, resulting in participation only if the benefit-cost ratio is higher than a certain threshold and such threshold increases with the probability of free-riding. Schmidt and Schnitzer (2003) show that increasing the number of OSS users by means of public subsidies can lead to an increase of software price proprietary software users who find themselves in a lock-in situation. Bitzer (2004) shows that product heterogeneity is the main factor explaining the ability of incumbent firm (producing PS) to be profitable by setting a higher price strategy than the new entrant producing OSS. Bessen (2004) shows that OSS production is more efficient because it satisfies more complex and sophisticated consumers' needs.

The second strand of literature adopts dynamic frameworks, by referring mainly to the role of increasing returns on the demand side. Such works introduced (both direct and indirect) network effects, which are likely to induce path-dependent processes (Arthur, 1989) and to produce lockin effects (David, 1985). In particular, Bonaccorsi and Rossi (2003) introduce an explicit OSS adoption function and show that, under some plausible assumptions, the two software production modes are likely to coexist. Dalle and Jullien (2003) introduce a more complex OSS adoption function by incorporating both local and global network effects. ${ }^{4}$ They find that the pace of code improvement and proselytism are important factors in explaining both OSS success and its coexistence with PS. In spite of their importance, these contributions still lack a formal analytical treatment that do not allow for general results.

Although developed within different frameworks (static models of industrial organization vs. non-linear dynamic models), all the models belonging to the two principal subsets reach a similar conclusion: PS and OSS are likely to coexist in the long-run. However, none of these works has properly analysed the dynamics of the diffusion of the two competing software, which are based on different industrial organization models. ${ }^{5}$

The literature on the diffusion of innovation is vast and covers different strands, from orthodox to heterodox ones. ${ }^{6}$

\footnotetext{
${ }^{4}$ The former refers to the proportion of a user's neighbours having already adopted OSS, the latter to the proportion of adopters in the whole population.

${ }^{5} \mathrm{~A}$ notable exception is the recent contribution of Casadesus-Masanell and Ghemawat (2006), who introduce a dynamic mixed duopoly model allowing competitors to have heterogeneous objective functions and model the presence of demand-side learning. Unlike the standard industrial organization models, in this paper the dynamics of competition between the two software are properly taken into account. However, differently from the approach of this paper, they do not consider (i) the supply side; (ii) :.:.:.:.:.:.:.; (iii)??????.

${ }^{6}$ For a comprehensive survey on models of technology diffusion reporting a set of different models' typologies see, for instance, Karshenas and Stoneman (1995) and Geroski
} 
In this paper, we therefore adopt an epidemic model of diffusion as it explains the diffusion dynamics in terms of a disequilibrium process during which knowledge comes to be differentially distributed among agents. In particular, we will follow the strand pioneered by Metcalfe (1981) and Batten (1987), who introduced a supply side in the demand-led model by Mansfield (1961). In this way, the dynamic path follows a logistic pattern determined by the joint dynamics of market demand and growth in production capacity. Further developments introduced more than one technique in order to show how the process of diffusion might be the result of competition among techniques, rather than the smooth diffusion of one only (Amable, 1992; Leoncini, 2001).

At the core of the epidemic approach there is the idea that the relevant features of a technology are not well known and, as the available information spreads, the level of uncertainty associated with it decreases, thus increasing the number of adopters. Expectations play a big role, as they come to depend on the information available from previous adopters, but also on the externalities gathered from the network of other users.

These characteristics are at the basis of our choice, since software adoption follows a path of progressive discovery of its main features, which is highly dependent on other adopters' information sets. As network externalities are heavily based on information exchange, this kind of models are appropriate, although some modifications are needed. Indeed, the analysis must take into account the peculiarities in software production and the process of competition involved. In particular, two major improvements are needed: (i) the presence of network effects on the demand side (a well known phenomenon discussed in the literature on network industries such as software, hardware, aircraft, etc., (Shy, 2001)); (ii) the possibility of joint adoption of competing products, which applies mainly to software industries characterised by increasing levels of standardization (Economides, 1996).

\section{General structure of the model}

In this Section we present the general structure of the model. Before doing this, some important stylised facts dealing with the task of modelling two competing software technologies using epidemic diffusion models must be addressed. In particular, five elements are worth discussing.

First of all, the diffusion of knowledge about (software) technologies can be compared to the diffusion of a disease, as they both take place through direct contact. Indeed, most of the time either a new operating system or a new application is likely to be adopted by a non-user if she is informed by a current user. Hence, the diffusion of knowledge about a technology can be thought as a disease that spreads all over a population of non-users

(2000). 
who, once infected, add up to the population of current users. This factor points to the adoption of epidemic models to model the process of interest as straightforward. ${ }^{7}$

Second, as already pointed out, epidemic models take into account the supply side by incorporating the production capacity growth rate (Metcalfe, 1981). This is a reasonable step to be taken if standard technologies are to be modelled. Nonetheless, our analysis concentrate on the software industry and here the relationship between demand and production capacity is not as strict as in other cases: in particular, software producers can instantaneously supply a new unit of output at negligible costs. This fact rules out the need to equate the rate of growth of demand and supply and their level at each point in time. Of course, accounting for the supply of software is important and we do it by relating it directly to the speed of technology diffusion, namely the propagation coefficient. Indeed, we assume that the two different modes of production characterising the two competing softwares impact directly on the probability that a user has to "infect" current non-users.

Third, several contributions have stressed the importance of, both positive and negative, network effects on the demand side as far as the software industry is concerned (Katz and Shapiro, 1985; Liebowitz and Margolis, 1994; Shy, 2001). Positive network externalities in the standard sense arise whenever "the utility that a user derives from consumption of a good increases with the number of other agents consuming the good" (Katz and Shapiro, 1985, p. 424). Although the standard models of epidemic diffusion are not micro-founded in terms of utility functions, we believe that the essence of the model is able to capture the effect of positive network externalities. Indeed, the logistic diffusion process for each technology implies that the probability for non-users to adopt the new technology is an increasing function of the population of those who have already adopted. Moreover, this effect, is not only based on a price mechanism, but it results in a true externality process (i.e. a word-of-mouth process).

We also takes into account the negative network externalities that likely arise from the need of interaction across users of different, and not perfectly inter-operable standards, when these different standards coexist in the market. Indeed, the fact that users of one software are forced to interact with users of other incompatible software reduce the utility they can get from it.

We model this feature by means of a parameter, $\eta(\geq 1)$ which captures the level of interoperability between the two software technologies. Moreover, because of the possible existence of asymmetries in this interoperabil-

\footnotetext{
${ }^{7}$ It must be underlined that we will not consider the possibility of differential morbidity among the population of adopters. Indeed, it could be possible to consider that OSS users behave differently from OSS developers. However, as the number of OSS adopters increases, the share of "simple" users increases so that we can assume that OSS and PS users tend to converge as the diffusion process unfolds.
} 
ity (Economides, 1996), we allow $\eta$ to be different across technologies. ${ }^{8}$ In particular, we denote with $\eta_{o p}$ the degree of interoperability of OSS (henceforth indicated by the $O$ subscript) with PS (henceforth indicated by the $P$ subscript), as it results from (i) the willingness of OSS developers to produce software inter-operable with PS, and (ii) the degree of closure of PS standards. This is because, in case of closed standards, it might still be possible for OSS developers to implement the standard by reverse engineering, but this requires more efforts and usually does not produce perfect results. Likewise, $\eta_{p o}$ denotes the interoperability level of PS with OSS, as it results mainly from the decisions of PS producers.

Fourth, switching costs are another feature peculiar of software industry. In general, switching costs arise when a buyer finds it costly to switch from one supplier to another because of habits produced by past purchasing decisions. In a market with switching costs a firm's current market share is therefore an important determinant of its future market success (Klemperer, 1995). We account for the possible existence of switching costs by means of a parameter, $\theta$, positively related with the extent of such costs and that measures the probability that an agent already using a technology does not adopt the other. Also in this case, we allow for possible differences in the extent of such costs: we thus denote with $\theta_{o p}$ the probability that a OSS user does not adopt a PS, and with $\theta_{p o}$ the probability that a PS user does not start using OS. Clearly, the larger the differences in the way the two technologies interact with the final user (languages, GUI, options, etc..), the greater the costs associated with the adoption of one technology for a previous user of the other. Moreover, the extent of this switching costs, and thus the value of $\theta$, may also be negatively related with the degree of interoperability $(\eta)$. Indeed, the lack of interoperability is a form of switching cost: since, when using the other software, one loses all the files and applications wrote in the old one. Hence, $\theta_{o p}$ should be properly intended has a function of $\eta_{o p}: \theta_{o p}=\theta_{o p}\left(\eta_{o p}, \gamma_{o}\right)$, where $\partial \theta_{o p} / \partial \eta_{o p}<0$. And the same should hold for $\theta_{p o}$, i.e. $\theta_{p o}=\theta_{p o}\left(\eta_{p o}, \gamma_{p}\right)$, with $\partial \theta_{p o} / \partial \eta_{p o}<0 .^{9}$

With this caveat in mind, $\theta$ enters into the model in two different ways: (i) the lower $\theta$, the higher the potential demand for a technology (since the probability that previous users of the other technology adopts it is greater); (ii) the lower $\theta$, the lower the negative across-technologies network effects given a certain degree of interoperability. Indeed, if the probability of users to move across technologies is quite high, one expects that some of the agents with whom she interacts will soon move to her technology and will prefer to

\footnotetext{
${ }^{8}$ For instance, OpenOffice can read and save files in Microsoft Office formats, whereas the latter does not recognise OpenDocument formats. In a similar manner, the great majority of Linux distributions can read and write file systems in the Microsoft's proprietary formats, while the opposite does not hold, unless by using third party packages.

${ }^{9} \mathrm{We}$ are indebted to one of the referees for this point.
} 
stay with the technology currently used rather than dismissing it. ${ }^{10}$

We assume that the maximum number of potential users of one or both the technologies $(D)$ is exogenously given. ${ }^{11}$ Each software technology diffuses following a logistic pattern. In particular, in line with the epidemic approach, we assume that every current adopter of PS $\left(A_{p}\right)$ in each instant in time has a given probability $\left(\beta_{p}\right)$ to induce the adoption of each non adopter $\left(D-A_{p}-A_{o}\right)$ plus each OSS user who is willing to adopt PS (given by $\left(1-\theta_{o p}\right) A_{o}$ ), which is negatively affected by the extent of switching costs). Moreover, the greater the fraction of OSS users not willing to adopt PS $\left(\theta_{o p} A_{o}\right)$, the greater the probability that each PS user will actually dismiss PS given the need for the PS users to interact with them when the degree of interoperability between PS and OSS $\left(\eta_{p o}\right)$ is actually small.

The diffusion dynamics of the number of PS adopters $\left(A_{p}\right)$ can thus be represented by the following non-linear differential equation:

$$
\dot{A}_{p}=\beta_{p} A_{p}\left(D-A_{p}-\theta_{o p} A_{o}\right)-\frac{\theta_{o p}}{\eta_{p o}} A_{o} A_{p}
$$

In a symmetric way, the diffusion dynamics of OSS adopters $\left(A_{o}\right)$ are given by:

$$
\dot{A}_{o}=\beta_{o} A_{o}\left(D-A_{o}-\theta_{p o} A_{p}\right)-\frac{\theta_{p o}}{\eta_{o p}} A_{p} A_{o}
$$

Despite the symmetry, the two technologies are characterised by two different production processes and system of incentives. PS is the product of standard profit-maximising firms, with high fixed costs and negligible marginal costs. On the contrary, OSS is produced thanks to the coordination of a community providing the source code for free. Hence, the most important factor influencing PS rate of diffusion is the price of the software while, for OSS, it is the effort lavished by the community of developers. This difference will be shown to entail different behaviours in the pattern of diffusion driven by differences in software qualities and characteristics.

As for OSS, it is always provided at no cost and for this reason its quality is initially low. Hence, the probability that potential users adopt OSS in

\footnotetext{
${ }^{10}$ It is also worth stressing that taking the levels of interoperability and switching costs as exogenous, as we do in what follows, does not exclude possible extensions where $\eta$ and $\theta$ result from endogenous and possibly strategic decisions of software producers. In this case, the comparative statics provided by the model can be still useful to look at the possible effects of the strategic choices. In this respect, an assumption of strategic behaviour seems more reasonable on the PS side than the OSS one, given the mostly decentralised structure behind the latter.

${ }^{11}$ Users do not have to be necessarily identical. Indeed, one might conceive our model as a sort of "mean-field" approximation when the agents are heterogeneous but fullymixed, so that they are distributed randomly in the population and their interactions do not exhibit strong clustering. Actually, when agents are highly heterogeneous in terms of preferences and capabilities and they interact through a fixed social network, the aggregate dynamics can be substantially more complex and may depend on the topology of the network (see, for instance, Vega-Redondo, 2007).
} 
following time steps is rather low. Nevertheless, OSS quality can improve, even at a high pace, if its user base increases. Indeed, some non adopters will be induced to choose OSS just because it is a free alternative to costly PS. On the other side, PS is characterised by a more constant quality in the sense that it comes to the market with a rather good quality level because the software house provides an already mature and tested product. However, in order to recoup the high development costs PS is initially characterised by a high selling price which hinders its pace of diffusion. When PS user base increases, the software house is able to spread fixed costs over a larger customer base, thus lowering the price. And this, in turn, increases further its speed of diffusion

The effectiveness of the word-of-mouth - the propagation coefficient $\beta$ in the two differential equations - is thus crucial and can actually be different for the competing technologies, since it turns out to be a function of the characteristics of the technology itself, which change in the diffusion.

In particular, as for PS, we assume that $\beta_{p}$ is a strictly decreasing and concave function of its price: $\beta_{p}=f_{p}(c)$ where $c$ is the price of PS, with $f_{p}^{\prime}(c)<0$ and $f_{p}^{\prime \prime}(c) \leq 0$. Moreover, given that software industry is characterised by economies of scale (Shy, 2001), we assume that the price of PS is a decreasing and convex function of its installed base: $c=c\left(A_{p}\right)$ with $c^{\prime}\left(A_{p}\right)<0$ and $c^{\prime \prime}\left(A_{p}\right)>0$. It follows that the speed of diffusion of PS turns out to be a strictly increasing and concave function of the number of PS users:

$$
\begin{gathered}
\beta_{p}=\beta_{p}\left(A_{p}\right)=f_{p}\left(c\left(A_{p}\right)\right) \\
\beta_{p}^{\prime}\left(A_{p}\right)=\frac{\mathrm{d} f_{p}(c)}{\mathrm{d} A_{p}}=f_{p}^{\prime}(c) c^{\prime}\left(A_{p}\right)>0 \\
\beta_{p}^{\prime \prime}\left(A_{p}\right)=\frac{\mathrm{d}^{2} f_{p}(c)}{\mathrm{d} A_{p}^{2}}=f_{p}^{\prime \prime}(c)\left(c^{\prime}\left(A_{p}\right)\right)^{2}+f_{p}^{\prime}(c) c^{\prime \prime}\left(A_{p}\right)<0
\end{gathered}
$$

As for OSS, given that its development does not entail any explicit monetary cost, but it is simply the result of the efforts made by the community responding both to intrinsic and extrinsic motivations (Lerner and Tirole, 2002; Bitzer et al., 2007), its final users do not face any direct explicit adoption cost, but only implicit ones. ${ }^{12}$

The OSS community should be conceived as an heterogeneous group of people willing to participate in the improvement of the software. This community is composed of different participants characterised by different levels of commitment to a particular project. We acknowledge the fact that developers have a large role to play in the process of creation of OSS code, we nevertheless believe that other typologies of participants (e.g. those

\footnotetext{
${ }^{12}$ It must be noted that some authors (e.g. Wheeler, 2005; Fitzgerald, 2006) acknowledge that the total cost of ownership, as implicit cost, may be as high as the explicit costs of buying a PS, although there is not unanimity on this point.
} 
occasionally supplying patches, reporting bugs, asking for assistance, etc.) have an important part in the process of ameliorating the final product (see Giuri et al. (2010) for empirical evidence on this point). ${ }^{13}$ For this reason, the effort of the community should be conceived as the sum of the individual efforts exerted by all of the typologies of participants to a particular OSS project. Relying on the fact that, the higher the effort of the community, the more the OSS is "developed" and therefore the less the costs borne by final users, we assume a negative relation between the total amount of such efforts and the level of these costs. The probability of OSS adoption $\left(\beta_{o}\right)$ is thus modelled as a strictly increasing function of community's efforts $(e)$ with non increasing marginal returns of efforts. In formal terms, $\beta_{o}=f_{o}(e)$, with $f_{o}^{\prime}(e)=0$ and $f_{o}^{\prime \prime}(e) \leq 0$.

Given that the sets of OSS final users and developers are likely to overlap due to the importance in the OSS method of production of user-driven innovation (see Section 2 and von Hippel and von Krogh (2003)), we assume that the level of efforts is positively related to the number of OSS adopters $\left(e^{\prime}\left(A_{o}\right)>0\right)$. Moreover, given that larger communities of developers are more likely to face coordination problems (such as, for instance, either disagreement on the actual piece of code to be incorporated into the final release, or disputes over credit attribution, with a higher probability of "forking" (Lerner and Tirole, 2002)), we assume further that the increase of efforts in development is less proportional than the increase of the level of adoption $\left(e^{\prime \prime}\left(A_{o}\right)<0\right) .^{14}$

Hence, the speed of diffusion of OSS can be represented as a strictly increasing and concave function of the number of OSS users:

$$
\begin{gathered}
\beta_{o}=\beta_{o}\left(A_{o}\right)=f_{o}\left(e\left(A_{o}\right)\right) \\
\beta_{o}^{\prime}\left(A_{o}\right)=\frac{\mathrm{d} f_{o}(e)}{\mathrm{d} A_{o}}=f_{o}^{\prime}(e) e^{\prime}\left(A_{o}\right)>0 \\
\beta_{o}^{\prime \prime}\left(A_{o}\right)=\frac{\mathrm{d}^{2} f_{o}(c)}{\mathrm{d} A_{o}^{2}}=f_{o}^{\prime \prime}(e)\left(e^{\prime}\left(A_{o}\right)\right)^{2}+f_{o}^{\prime}(e) e^{\prime \prime}\left(A_{o}\right)<0
\end{gathered}
$$

Overall, although the production of PS and OSS is driven by different types of actors, firms and community of developers respectively, which respond to different types of incentives, $c$ and $e$ respectively, and, thus, are eminently different in their intimate essence, the effects we derive on the

\footnotetext{
${ }^{13}$ The individual effort can be conceived as the opportunity cost of choosing to participate to the community. This cost is evidently higher for core developers compared to less technical contributors, but core developers are less numerous compared to other kinds of participants. So, we expect the aggregate level of effort to represent a more balanced mixture of developers and final users. Based on that, the effort level is expected to increase with the OSS user base.

${ }^{14}$ Strong evidence supporting this assumption has been recently provided by an empirical work employing data on the population of OSS projects hosted on SourceForge.net (Comino et al., 2007).
} 
relationship between speed of diffusion and level of adoption turn out to be similar for both software. It is worth noting that our model is able to report differences in behaviour without an explicit functional forms of the different propagation coefficients $\left(\beta_{p}\right.$ and $\left.\beta_{o}\right)$. This is an advantage, as by making reasonable assumptions on the first and second derivatives only, we provide a tractable model of such a complex process of diffusion.

The dynamics of diffusion can be thus represented by the following autonomous non-linear system of differential equations:

$$
\begin{aligned}
& \dot{A}_{p}=\beta_{p}\left(A_{p}\right) A_{p}\left(D-A_{p}-\theta_{o p} A_{o}\right)-\frac{\theta_{o p}}{\eta_{p o}} A_{o} A_{p} \\
& \dot{A}_{o}=\beta_{o}\left(A_{o}\right) A_{o}\left(D-A_{o}-\theta_{p o} A_{p}\right)-\frac{\theta_{p o}}{\eta_{o p}} A_{p} A_{o}
\end{aligned}
$$

The system reduces to a standard Lotka-Volterra model for two competing species (Hofbauer and Sigmund, 1998) when $\beta$ is constant and $\eta \rightarrow \infty$ for both technologies. We innovate this baseline model in three respects: (i) we model the supply of software, being it PS or OSS, by endogenising the propagation coefficient; (ii) we introduce negative network effects on the demand side (positive ones are already considered in the logistic diffusion process); (iii) we account for the effect of switching costs on both the share of would-be adopters and the extent of network effects.

\section{Diffusion patterns with constant propagation co- efficient}

In this Section, we discuss the results of the model by assuming that the actual level of technology diffusion does not significantly affect the relevant features of the technology (i.e. the price for PS and the level of development for OSS $\left.\left(c^{\prime}\left(A_{p}\right)=e^{\prime}\left(A_{o}\right)=0\right)\right)$; or, equivalently, that such features do not alter the probability of adoption $\left.\left(f_{p}^{\prime}(c)=f_{o}^{\prime}(e)=0\right)\right)$. Thus, we have that $\beta_{p}$ and $\beta_{o}$ are constant, and system (5) can be written:

$$
\begin{aligned}
& \dot{A}_{p}=\beta_{p} A_{p}\left(D-A_{p}-\left(1+\frac{1}{\eta_{p o} \beta_{p}}\right) \theta_{o p} A_{o}\right) \\
& \dot{A}_{o}=\beta_{o} A_{o}\left(D-\left(1+\frac{1}{\eta_{o p} \beta_{o}}\right) \theta_{p o} A_{p}-A_{o}\right)
\end{aligned}
$$

Therefore, with a constant propagation coefficient, equations (6) replicate the well-known Lotka-Volterra equations for two competing species (see, for instance, Hofbauer and Sigmund, 1998, Ch.3). The isoclines are straight 
lines with negative slopes:

$$
\begin{aligned}
A_{o} & =\frac{D}{\left(1+\frac{1}{\eta_{p o} \beta_{p}}\right) \theta_{o p}}-\frac{1}{\left(1+\frac{1}{\eta_{p o} \beta_{p}}\right) \theta_{o p}} A_{p} \\
A_{o} & =D-\left(1+\frac{1}{\eta_{o p} \beta_{o}}\right) \theta_{p o} A_{p}
\end{aligned}
$$

Given the constraints on the parameters, these lines intersect at most once provided that either $\eta_{p o} \beta_{p} \neq \theta_{o p} /\left(1-\theta_{o p}\right)$ or $\eta_{o p} \beta_{o} \neq \theta_{p o} /\left(1-\theta_{p o}\right)$. Figure 1 depicts the possible cases with the out of equilibrium directions.

The sufficient and necessary condition for the stable coexistence of the technologies in the market is therefore that the following inequalities hold: ${ }^{15}$

$$
\eta_{p o} \beta_{p}>\frac{\theta_{o p}}{1-\theta_{o p}} \quad \eta_{o p} \beta_{o}>\frac{\theta_{p o}}{1-\theta_{p o}}
$$

i.e. the speed of adoption corrected for the interoperability degree must be greater than the odds against the adoption of the technology by the current users of the other technology (Figure 1(a)).

If condition (9) holds for only one technology, this technology displaces completely the other (Figures 1(c) and 1(d)). When instead condition (9) does not hold for any technology, we are in the so called bistable case (Figure $1(b))$. There are two basins of attraction: the orbits in the first one converge to $(D, 0)$, whereas the others to $(0, D)$, while $E$ is a saddle point. In such a case, initial conditions matter.

Let us note that, in case of stable coexistence, a decrease of the switching costs to one technology for previous users of the other technology decreases the total number of users of that technology, both exclusive users and cousers; whereas an increase of the interoperability of one technology actually makes these users increase. In formal terms, if condition (9) is satisfied and there are some switching costs $(\theta>0)$ for both technologies, we have the following set of partial derivatives:

$$
\begin{array}{llll}
\frac{\partial A_{p}^{*}}{\partial \theta_{o p}}<0 & \frac{\partial A_{o}^{*}}{\partial \theta_{o p}}>0 & \frac{\partial A_{o}^{*}}{\partial \theta_{p o}}<0 & \frac{\partial A_{p}^{*}}{\partial \theta_{p o}}>0 \\
\frac{\partial A_{p}^{*}}{\partial \beta_{p}}>0 & \frac{\partial A_{o}^{*}}{\partial \beta_{p}}<0 & \frac{\partial A_{o}^{*}}{\partial \beta_{o}}>0 & \frac{\partial A_{p}^{*}}{\partial \beta_{o}}<0 \\
\frac{\partial A_{p}^{*}}{\partial \eta_{p o}}>0 & \frac{\partial A_{o}^{*}}{\partial \eta_{p o}}<0 & \frac{\partial A_{o}^{*}}{\partial \eta_{o p}}>0 & \frac{\partial A_{p}^{*}}{\partial \eta_{o p}}<0 .
\end{array}
$$

\footnotetext{
${ }^{15}$ Let us note that this equilibrium is globally stable (or uniformly asymptotically stable in the large). Thus, the initial conditions do not actually matter. For a proof of the global stability of the equilibrium in the case of stable coexistence for the Lotka-Volterra equations for two competing species by means of the Lyapunov function see, for instance, Medio and Lines (2001).
} 

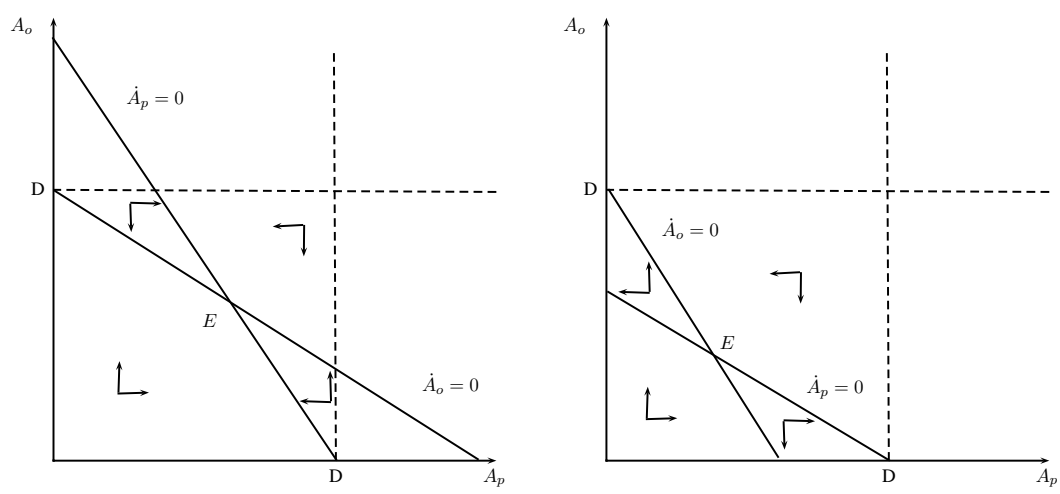

(a) Asymptotically stable equilibrium (b) Saddle point (low interoperabilwith technologies' coexistence (high interoperability/low switching costs for both)
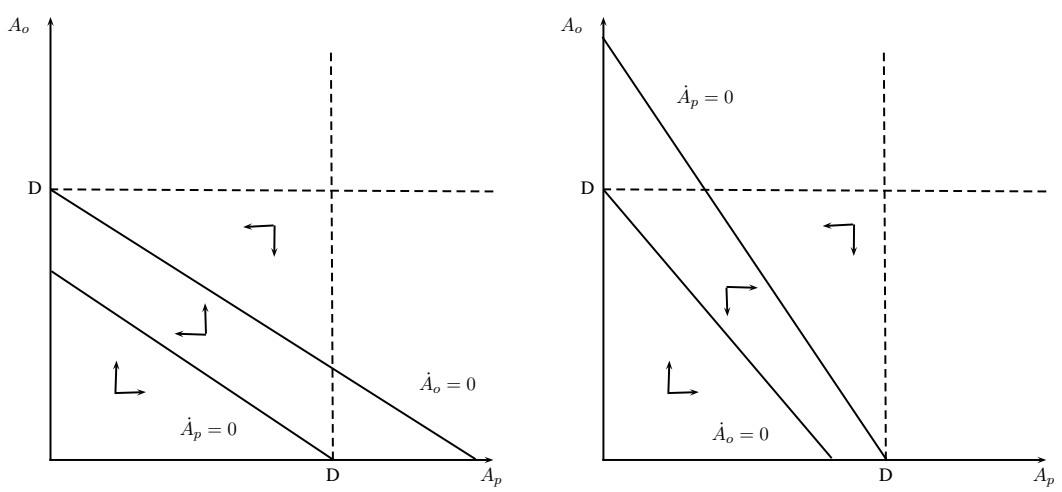

(c) Market tips in favor of OSS (low (d) Market tips in favour of PS (low interoperability of PS with OSS/high interoperability of OSS with PS/high cost of switching from OS to PS) cost of switching from PS to OSS)

Figure 1: Dynamics with constant propagation coefficient
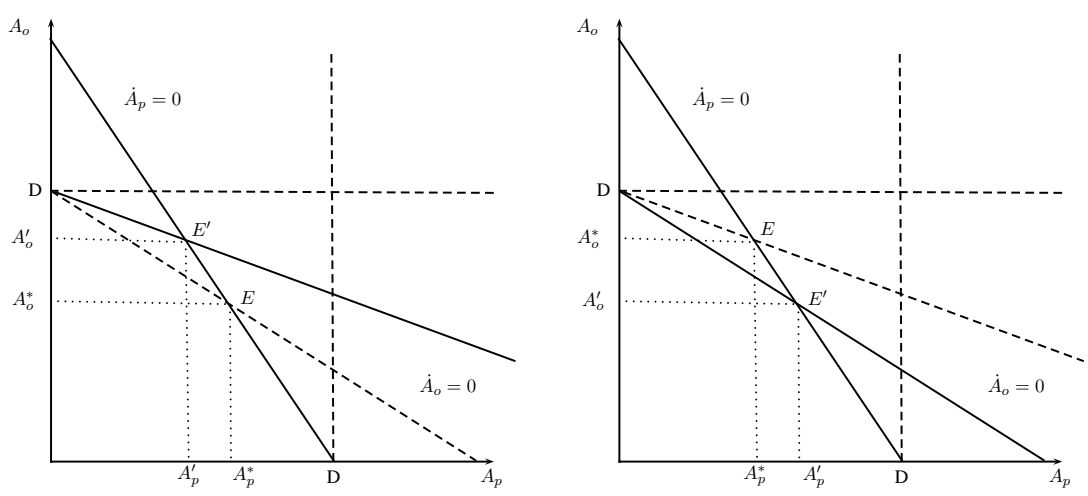

(a) Decrease in the costs of switching (b) Closure of PS standards ( $\eta_{o p}$ defrom PS to OSS ( $\theta_{p o}$ decreases) creases)

Figure 2: Comparative statics 
So, for instance, when the costs of switching from PS to OSS $\left(\theta_{p o}\right)$ decreases, there is an absolute increase of OSS users (from $A_{o}^{*}$ to $A_{o}^{\prime}$ in Figure 2(a)) and a decrease of PS users (from $A_{p}^{*}$ to $A_{p}^{\prime}$ in Figure 2(a)).

On the contrary, if the degree of interoperability of OSS technology decreases because of the closure of the standards adopted by PS, this makes total OSS users decrease, whereas PS users instead increases (Figure 2(b)). Thus, the closure of the standard by the PS producer is a very effective strategy in order to tip the market, causing a more than proportional decrease in OSS market share. ${ }^{16}$

When $\eta \beta \gg 1$, the number of users who will jointly use the two technologies in equilibrium in case of stable coexistence will be $\left(1-\left(1+\frac{1}{\eta_{p o} \beta_{o}}\right) \theta_{o p}\right) A_{o}^{*}$ $\left(=\left(1-\left(1+\frac{1}{\eta_{o p} \beta_{p}}\right) \theta_{p o}\right) A_{p}^{*}\right)$.

Let us finally note that the market shares in case of stable coexistence are not affected by the absolute size of the market. ${ }^{17}$

\section{Diffusion patterns with changing propagation coefficient}

In this Section we modify the previous model by assuming that the two different supply structures influence the propagation coefficient and, through it, the patterns of diffusion. In particular, Section 5.1 assumes perfect interoperability between the two technologies, whereas Section 5.2 takes into account the most complex case of the diffusion of two competing software technologies with non-perfect interoperability and switching costs.

\subsection{Perfect interoperability}

In the limiting case of perfect interoperability (i.e. $\eta_{p o} \rightarrow \infty$ and $\eta_{o p} \rightarrow \infty$ ), the system of differential equations (5) becomes:

$$
\begin{aligned}
& \dot{A}_{p}=\beta_{p}\left(A_{p}\right) A_{p}\left(D-A_{p}-\theta_{o p} A_{o}\right) \\
& \dot{A}_{o}=\beta_{o}\left(A_{o}\right) A_{o}\left(D-A_{o}-\theta_{p o} A_{p}\right)
\end{aligned}
$$

\footnotetext{
${ }^{16}$ However, it is worth noting that, by taking into account the possible impact that the degree of interoperability has on the costs of switching, the final result can be less sharp. Indeed, when the decrease of $\eta_{o p}$ produces an increase in $\theta_{o p}$, in addition to the clockwise rotation of the isocline of $A_{o}$ showed in the Figure 2(b), the isocline of $A_{p}$ rotates counterclockwise around the point $\mathrm{D}$ on the abscissa. This will tend to reduce the number of co-users and thus reduces both the number of total users of $A_{o}$ and $A_{p}$. The final effect will depend on the relative strength of these two effects.

${ }^{17}$ The ratio between the total number of OS and PS users is indeed given by

$$
\frac{A_{o}^{*}}{A_{p}^{*}}=\frac{\eta_{p o} \beta_{p}}{\eta_{o p} \beta_{o}} \cdot \frac{\theta_{p o}-\eta_{o p} \beta_{o}\left(1-\theta_{p o}\right)}{\theta_{o p}-\eta_{p o} \beta_{p}\left(1-\theta_{o p}\right)}
$$
}

and it does not depend on the total amount of demand $(D)$. 
Although $\beta$ is now a function of the actual level of diffusion of the relative technology, the equilibrium values depend only on the extent of switching $\operatorname{costs}(\theta)$ and are equal to:

$$
\begin{aligned}
A_{p}^{*} & =\frac{1-\theta_{o p}}{1-\theta_{o p} \theta_{p o}} D \\
A_{o}^{*} & =\frac{1-\theta_{p o}}{1-\theta_{o p} \theta_{p o}} D
\end{aligned}
$$

Provided that these parameters are not degenerate, such equilibrium is asymptotically stable, no matter what the actual forms of the functions $\beta$ are (see Appendix A.1 for a proof of the asymptotic stability of the equilibrium). In such case, the situation is still the one depicted in Figure 1(a), although now there is perfect interoperability. Thus, in the present case the outcome is always the stable coexistence.

It is worth stressing that the market shares of the competing technologies are not affected by those features that interact with the propagation coefficient (i.e. the price for PS and level of development for OSS), but only by the ones which instead affect the switching costs. Thus, by assuming perfect interoperability and the presence of switching costs, the final outcome is neither the most efficient one nor the one in which the product with the best features (effective or potential) is actually chosen (Arthur, 1989). In the stable equilibrium all the users adopt at least one technology, whereas the number of users who jointly adopt the two is equal to $\left(1-\theta_{o p}\right) A_{o}^{*}(=$ $\left.\left(1-\theta_{p o}\right) A_{p}^{*}\right)$.

\subsection{Non-perfect interoperability}

In order to analyse the dynamics in the most complex case, we work out the isoclines:

$$
\begin{aligned}
A_{o}^{*}\left(A_{p}\right) & =\frac{1}{\theta_{o p}} \frac{\eta_{p o} \beta_{p}\left(A_{p}\right)}{1+\eta_{p o} \beta_{p}\left(A_{p}\right)}\left(D-A_{p}\right) \\
A_{p}^{*}\left(A_{o}\right) & =\frac{1}{\theta_{p o}} \frac{\eta_{o p} \beta_{o}\left(A_{o}\right)}{1+\eta_{o p} \beta_{o}\left(A_{o}\right)}\left(D-A_{o}\right)
\end{aligned}
$$

Let us note first that the convex hull of $\{(0,0),(D, 0),(D, D),(0, D)\}$ is the only relevant area, given that the threshold $D$ is a physical constraint (i.e. the actual number of users of each technology cannot be greater than the maximum feasible number of users). Hence, we have to take into account only the interval $[0, D]$ for each variable.

To start with, we analyse the isocline of $A_{p}$ in such interval. For equation (12), we have:

$$
\frac{\mathrm{d} A_{o}^{*}}{\mathrm{~d} A_{p}}=\frac{\eta_{p o} \beta_{p}\left(A_{p}\right)}{\theta_{o p}\left(1+\eta_{p o} \beta_{p}\left(A_{p}\right)\right)^{2}}\left(\frac{\beta_{p}^{\prime}\left(A_{p}\right)}{\beta_{p}\left(A_{p}\right)}\left(D-A_{p}\right)-\left(1+\eta_{p o} \beta_{p}\left(A_{p}\right)\right)\right)
$$




$$
\frac{\mathrm{d}^{2} A_{o}^{*}}{\mathrm{~d} A_{p}^{2}}=-\frac{2 \eta_{p o}\left(D-A_{p}\right)}{\theta_{o p}\left(1+\eta_{p o} \beta_{p}\left(A_{p}\right)\right)^{2}}\left(\frac{\eta_{p o} \beta_{p}^{\prime}(x)^{2}}{1+\eta_{p o} \beta_{p}(x)}+\frac{\beta_{p}^{\prime}(x)}{D-x}-\frac{\beta_{p}^{\prime \prime}(x)}{2}\right)
$$

If conditions (1) and (2) hold and $\theta_{o p}$ is not degenerate, we have $\mathrm{d}^{2} A_{o}^{*} / \mathrm{d} A_{p}^{2}<$ 0 for $A_{p} \in[0, D]$. Hence, equation (12) is strictly concave in such interval. Moreover, given that:

$$
\left.\frac{\mathrm{d} A_{o}^{*}}{\mathrm{~d} A_{p}}\right|_{A_{p}=D}=-\frac{1}{\theta_{o p}} \frac{\eta_{p o} \beta_{p}(D)}{1+\eta_{p o} \beta_{p}(D)}(<0)
$$

and $\lim _{A_{p} \rightarrow D} A_{o}^{*}\left(A_{p}\right)=0$, by the strict concavity of $A_{o}^{*}\left(A_{p}\right)$ follows that:

$$
A_{o}^{*}\left(A_{p}\right)=A_{o}^{*}\left(D+\left(A_{p}-D\right)\right)<\frac{1}{\theta_{o p}} \frac{\eta_{p o} \beta_{p}(D)}{1+\eta_{p o} \beta_{p}(D)}\left(D-A_{p}\right)
$$

for each $A_{p} \in[0, D)$. Thus, the function $A_{o}^{*}\left(A_{p}\right)$ lies below the straight line:

$$
A_{o}=\frac{\eta_{p o} \beta_{p}^{M} D}{\left(1+\eta_{p o} \beta_{p}^{M}\right) \theta_{o p}}-\frac{\eta_{p o} \beta_{p}^{M}}{\left(1+\eta_{p o} \beta_{p}^{M}\right) \theta_{o p}} A_{p}
$$

where $\beta_{p}^{M}=\beta_{p}(D)$. This line is the isocline of $A_{p}$ in the model with a constant propagation coefficient, calculated at the maximum attainable PS propagation coefficient (equation (7)).

Moreover, by assuming $\lim _{A_{p} \rightarrow 0} \beta_{p}\left(A_{p}\right)=0$, we have:

$$
\lim _{A_{p} \rightarrow 0} \frac{\mathrm{d} A_{o}^{*}}{\mathrm{~d} A_{p}}=\frac{\eta_{p o} \beta_{p}^{\prime}(0)}{\theta_{o p}} D>0
$$

and there is therefore a unique local maximum of the function $\left(A_{o M}^{*} \in\right.$ $\left.\left[0, \frac{D}{\theta_{o p}}\right]\right)$ lying in the domain $(0, D){ }^{18}$ Hence, the function is as in Figure $3(\mathrm{a})$ and $A_{p}$ will increase (decrease) depending on the combination $\left(A_{p}, A_{o}\right)$ being actually below (above) the function.

What remains to be analysed are the effects of the two parameters $\left(\theta_{o p}\right.$ and $\eta_{p o}$ ) on the shape of the function. As for $\theta_{o p}$, it is sufficient to note that it enters the function simply as a multiplicative constant. Hence, an increase of $\theta_{\text {op }}$ moves $A_{p}$ isocline downward as in Figure 4(a). As for $\eta_{p o}$, an increase of it makes the curve change as in Figure 4(b) (see Appendix B for a more in depth analysis of the effects of the parameters on $A_{o}^{*}(x)$ ).

\footnotetext{
${ }^{18}$ When $\eta_{p o} \rightarrow \infty$ we have:$$
\lim _{A_{p} \rightarrow 0} A_{o}^{*}\left(A_{p}\right)=\lim _{A_{p} \rightarrow 0} \frac{\beta_{p}\left(A_{p}\right) D}{\theta_{o p} \beta_{p}\left(A_{p}\right)}=\lim _{A_{p} \rightarrow 0} \frac{\beta_{p}^{\prime}\left(A_{p}\right) D}{\theta_{o p} \beta_{p}^{\prime}\left(A_{p}\right)}=\frac{D}{\theta_{o p}} .
$$$$
\lim _{A_{p} \rightarrow 0} \frac{\mathrm{d} A_{o}^{*}}{\mathrm{~d} A_{p}}=\lim _{A_{p} \rightarrow 0}-\frac{\beta_{p}\left(A_{p}\right)^{2}}{\theta_{o p} \beta_{p}\left(A_{p}\right)^{2}}=\lim _{A_{p} \rightarrow 0}-\frac{\beta_{p}^{\prime}\left(A_{p}\right)\left(\beta_{p}^{\prime}\left(A_{p}\right)+\beta_{p}^{\prime \prime}\left(A_{p}\right)\right)}{\theta_{o p} \beta_{p}^{\prime}\left(A_{p}\right)\left(\beta_{p}^{\prime}\left(A_{p}\right)+\beta_{p}^{\prime \prime}\left(A_{p}\right)\right)}=-\frac{1}{\theta_{o p}}
$$

and we are back in the case analysed in Section 5.1.
} 


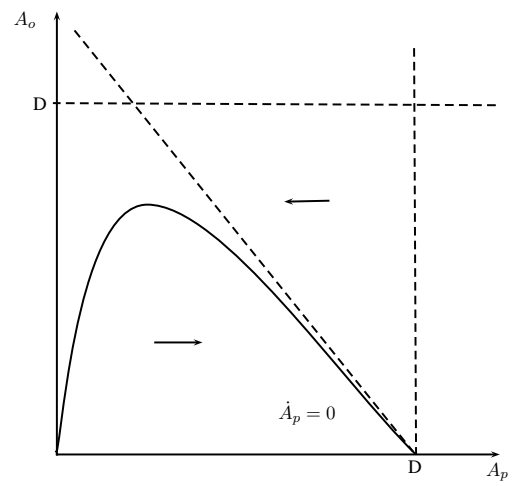

(a) Isocline of $A_{p}$

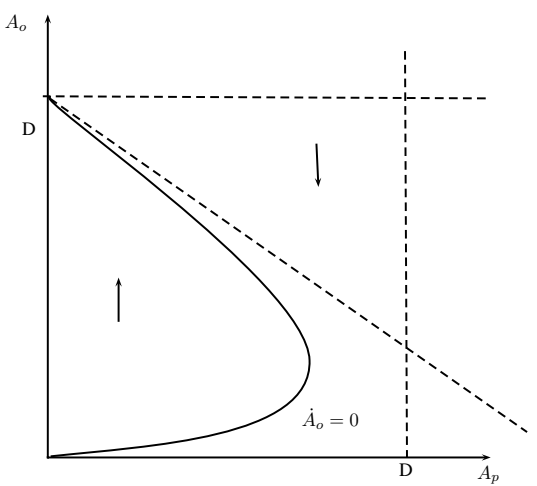

(b) Isocline of $A_{o}$

Figure 3: Isoclines

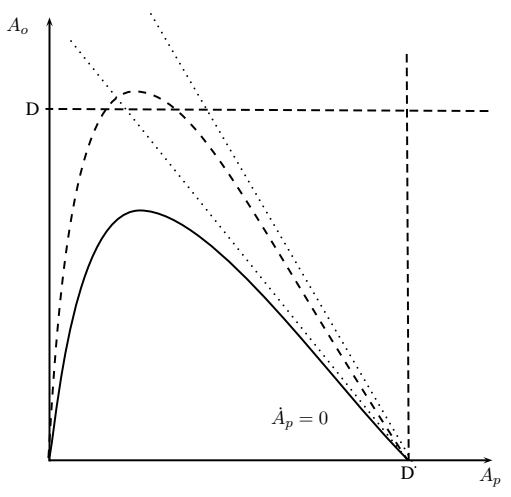

(a) Increase of $\theta_{o p}$

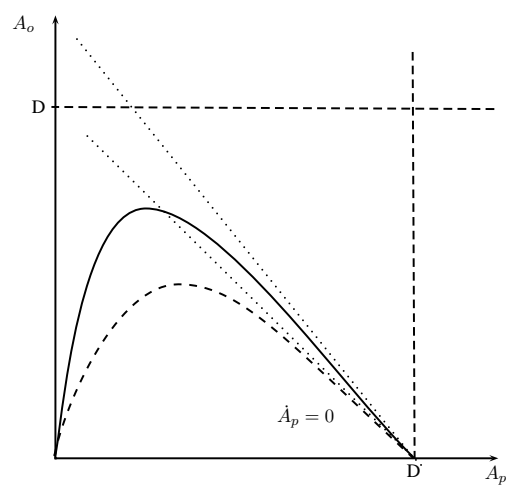

(b) Increase of $\eta_{p o}$

Figure 4: Changes of the parameters

In the light of the analogy of assumptions (1)-(2) and (3)-(4), equations (12) and (13) are symmetric with respect to the axes and the isocline of $A_{o}$ is the one shown in Figure 3(b). All the analysis carried out for the isocline of $A_{p}$ is therefore valid also for the other isocline provided that the notation for $A_{p}$ is substituted with the notation for $A_{o}$.

The solutions for the most complex case are depicted in Figure 5. We can have one stable solution with coexistence of both technologies (point $E$ in Figure $5(\mathrm{a})),{ }^{19}$ which is not a globally stable equilibrium, as points outside the hearth-shaped area tend towards equilibria characterised by winner-takeall solutions (points $D$ ). The stability of the equilibrium point depends on the shape of the isoclines. Indeed, if the value of $\theta$ is big enough and/or that of $\eta$ is small enough, the area of stable trajectories shrinks, and the equilibrium point $E$ becomes a saddle point (Figure $5(\mathrm{~b})$ ), which shows one

\footnotetext{
${ }^{19}$ See Appendix A.2 for a proof of the stability of the equilibrium.
} 
winning technology and dependence from initial conditions .

These last features of the model, allow to make some interesting comparative dynamics exercises (Figure 6), to introduce, although in a very crude way, some strategic interactions. First, a closure of the PS standard is likely to induce a strong negative effect on OSS that can even tip the market in favor of PS (Figure 6(a)). This implies that PS producers have an important strategic instrument to reduce the degree of interoperability of OSS and lock-in the market towards proprietary technology. ${ }^{20}$ Second, a decrease in the level of switching costs for PS users produces an increase in the overall number of users adopting OSS (Figure 6(b)). This increase can be a consequence of the commitment of OSS towards user-friendly applications and the development of graphical interfaces similar to existing PS ones.

It should also be noted that, as far as OSS is concerned, the community ethos (i.e. the strong sense of belonging to the community of developers) can decrease considerably the probability of OSS users to adopt PS. This can be regarded as a sort of "psychological" switching costs that increase $\theta_{\text {op }}{ }^{21}$

Finally, it might happen that, with a low level of interoperability for both technologies, no diffusion actually takes place (Figure 6(c)). Nevertheless, in this case the more likely outcome is that the technology characterised by even a small advantage locks the market in.

A final remark is on the possibility that, besides the negative externalities across technologies related to the lack of interoperability analyzed in the model, there might be positive externalities not only within technologies, but also across technologies. Indeed, in case of compatible software, the adopters of one software may enjoy of a larger installed base of the other software given that they can exchange files and information with them. ${ }^{22}$ Appendix $\mathrm{C}$ analyze a simple extension of the model to account for this possibility and somehow show that the main results we derived still hold.

\section{Conclusions}

This paper has shown how to implement a formal model of innovation diffusion to model two competing technologies in high-tech industries. Indeed, as they are characterised by the presence of economies of scale, within- and across-technologies network effects and switching costs, a proper theoretical

\footnotetext{
${ }^{20}$ It is worth noting that the probability of such complete displacement is actually reduced when the decreased interoperability produces an increase of switching costs of OSS users to PS. Indeed, the increase of $\theta_{o p}$ generated by the decrease of $\eta_{o p}$ actually moves down the isocline of $A_{p}$ and a new stable equilibrium with coexistence can arise.

${ }^{21}$ In this sense, for example, the diffusion of the Free Software movement and the creation of Free Software Foundation (1984), along with the strong charisma of his founder, Richard Stallman, have been a great vehicle of diffusion.

${ }^{22} \mathrm{We}$ acknowledge one of the referees for this remark.
} 


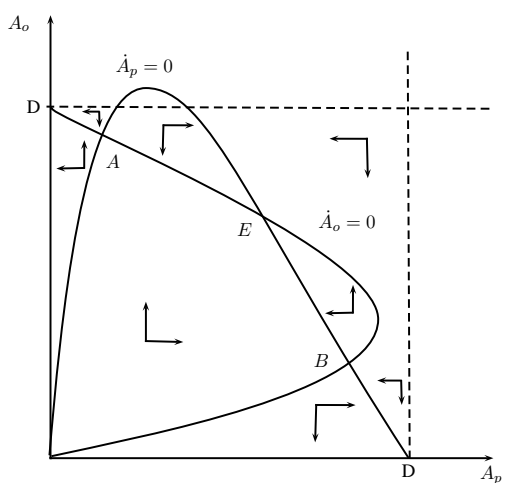

(a) Stable node

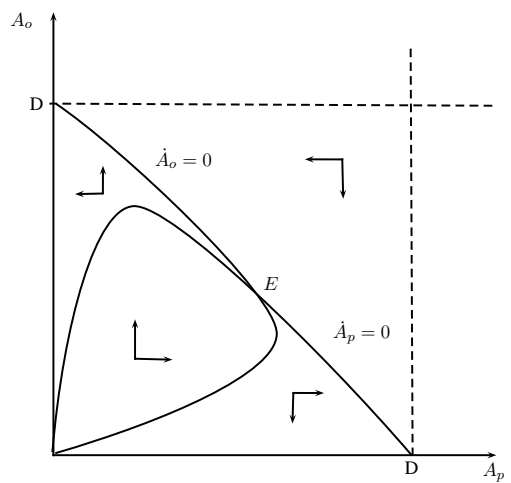

(b) Saddle point

Figure 5: Fixed points
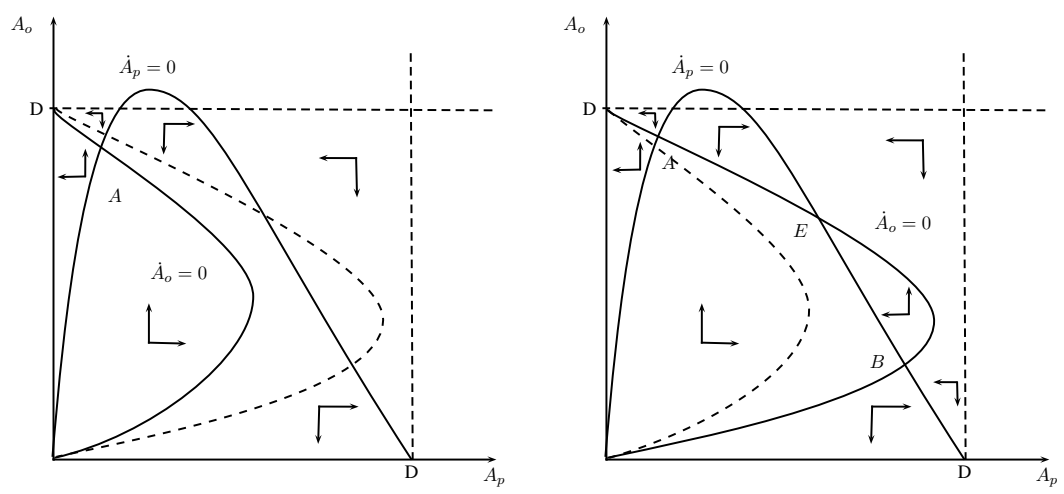

(a) Decrease of OSS interoperability (b) Decrease of switching costs for PS $\left(\eta_{o p}\right)$ users $\left(\theta_{p o}\right)$

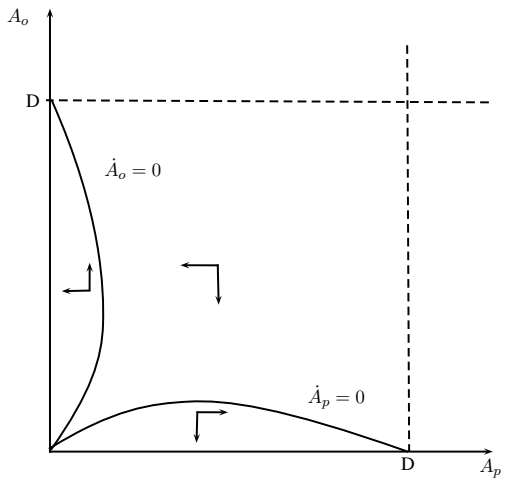

(c) Strong lack of interoperability

Figure 6: Comparative dynamics 
modelling is needed, that, to our knowledge, has not been carried out so far. Moreover, when open-source is considered, other issues arise, such as the effort of the community and developers' motivations.

All these topics have been incorporated in a modified version of a standard epidemic model, which innovate the existing literature in some respects. Above all, the endogenisation of the propagation coefficient adds substantial realism to the general structure of the model, yielding interesting results, such as the coexistence of an asymptotically stable equilibrium where both technologies survive with winner-take-all solutions. This result is obtained in a dynamic setting, thus enriching the achievements of the literature on OSS-PS competition, which obtained it only in a static context. Moreover, our model adds up new insights to the literature on the diffusion of competing technologies under increasing returns where the standard result is that the market tips in favour of one of the two. Indeed, the process of competition between technologies has been modelled by the literature as a situation where either one technology tips the market for an indefinite period of time (David, 1985; Arthur, 1989; Amable, 1992) or a superior technology, after being adopted by a critical mass of users, displaces the other one (Witt, 1997; Andreozzi, 2004). On the contrary, we show how both coexistence and market tipping are likely outcomes also in a situation where both technologies start the competition process at the same time. Finally, network effects and switching costs turn out to be important factors that the supplier of one technology can change in order to alter the equilibrium point and thus its market share.

Within this innovative theoretical framework, the main results are the following. First of all, in all the different specifications of the model there is always the possibility to obtain an asymptotically stable equilibrium where both technologies coexist. Thus, contrary to the result obtained by Amable (1992), the process of competition between two technologies characterised by increasing returns do not necessarily ends up with one of the two tipping the market, at least in the present case, where the possibility of joint adoption is taken into account.

Second, we have been able to present the conditions that determine the success of one technology with respect to another one: (i) under the assumption of a constant propagation coefficient, the probability of adoption corrected for the interoperability degree must be greater than the odds against the adoption of the technology by the current users of the other technology; (ii) when the coefficient of propagation is let to vary, then the condition can be computed formally only if a functional form for the propagation coefficient is assumed.

Third, in the case of coexistence of both technologies, it is possible to modify the equilibrium by changing the values of the parameters $\theta$ and $\eta$. In particular, a decrease of the switching costs for PS users $\left(\theta_{p o}\right)$ yields a more than proportional increase in the number of OSS users and a simultaneous 
decrease in the absolute number of PS users. If the interoperability of OSS $\left(\eta_{o p}\right)$ decreases, thus increasing the negative network effects produced by the mass of PS users, then the number of OSS users decreases and PS users increase. However, such effect can be counterbalanced by the opposite effect created by the consequential increase of the costs of switching for OSS users $\left(\theta_{\text {op }}\right){ }^{23}$

Finally, the stability of the equilibrium point is a recursive result through the different specifications of the model. However, while under the assumption of exogenous probability of adoption, the equilibrium point is globally stable, when the propagation coefficient is endogenous the equilibrium point is only locally stable and it coexists with two other points around which one of the two technologies is likely to tip the market. In this last case, a change in the parameters $\theta$ and $\eta$ leads to a modification of the basin of attraction of the equilibrium point.

\section{References}

Amable, B. (1992) Competition among techniques in the presence of increasing returns to scale, Journal of Evolutionary Economics, 2, pp. 147-158.

Andreozzi, L. (2004) A note on critical masses, network externalities and converters, International Journal of Industrial Organization, 22(5), pp. 647-653.

Arthur, B. (1989) Competiting technologies, increasing returns, and lock-in by historical events, The Economic Journal, 99(394), pp. 116-131.

Batten, D. (1987) The balanced path of economic development: a fable for growth merchants, in: D. Batten, J. Casti and B. Johansson (Eds.) Economic Evolution and Structural Adjustment (Springer-Verlag).

Bessen, J. (2004) Open Source Software: Free Provision of Complex Public Goods, Working paper, SSRN.

Bitzer, J. (2004) Commercial versus open source software: the role of product heterogeneity in competition, Economic Systems, 28(4), pp. 369-381.

Bitzer, J., Schrettl, W. and Schroeder, P. J. (2007) Intrinsic motivation in open source software development, Journal of Comparative Economics, 35(1), pp. 160-169.

Bonaccorsi, A. and Rossi, C. (2003) Why open source software can succeed, Research Policy, 32(7), pp. 1243-58.

\footnotetext{
${ }^{23}$ These are mainly comparative statics' exercises and not a complete strategic analysis which constitutes food for thought of a future paper.
} 
Brynjolfsson, E., Kemerer, C. and of Management, S. S. (1996) Network Externalities in Microcomputer Software: An Econometric Analysis of the Spreadsheet Market, Management Science, 42, pp. 1627-1647.

Casadesus-Masanell, R. and Ghemawat, P. (2006) Dynamic mixed duopoly: A model motivated by linux vs. windows, Management Science, 52(7), pp. 1072-1084.

Chiaravutthi, Y. (2006) Firms'strategies and network externalities: Empirical evidence from the browser war, Journal of High Technology Management Research, 17(1), pp. 27-42.

Comino, S., Manenti, F. and Parisi, M. (2007) From planning to mature: On the success of open source projects, Research Policy, 36(10), pp. 15751586 .

Dalle, J. M. and Jullien, N. (2003) 'libre' software: Turning fads into institutions?, Research Policy, 32(1), pp. 1-11.

David, P. (1985) Clio and the economics of QWERTY, American Economic Review: Papers and Proceedings, 75, pp. 332-337.

Economides, N. (1996) The economics of networks, International Journal of Industrial Organization, 14(6), pp. 673-699.

Fitzgerald, B. (2006) The Transformation of Open Source Software, Management Information Systems Quarterly, 30(3), p. 587.

Fosfuri, A., Giarratana, M. and Luzzi, A. (2008) The penguin has entered the building: The commercialization of open source software products, Organization Science, 19(2), p. 292.

Gandal, N. (1994) Hedonic price indexes for spreadsheets and an empirical test for network externalities, RAND Journal of Economics, 25(1), pp. 160-170.

Gandal, N. (1995) Competing compatibility standards and network externalities in the pc software market, The Review of Economics and Statistics, 77(4), pp. 599-608.

Geroski, P. A. (2000) Models of technology diffusion, Research Policy, 29(45), pp. 603-625.

Giuri, P., Ploner, M., Rullani, F. and Torrisi, S. (2010) Skills, division of labor and performance in collective inventions: Evidence from open source software, International Journal of Industrial Organization, 28(1), pp. 5468. 
Hofbauer, J. and Sigmund, K. (1998) Evolutionary Games And Population Dynamics (Cambridge: Cambridge University Press).

Johnson, J. P. (2002) Open source software: Private provision of a public good, Journal of Economics $\& 3$ Management Strategy, 11(4), pp. 637-662.

Karshenas, M. and Stoneman, P. (1995) Technological diffusion, in: P. Stoneman (Ed.) Handbook of the Economics of Innovation and Technical Change (Basil Blackwell).

Katz, M. L. and Shapiro, C. (1985) Network externalities, competition, and compatibility, American Economic Review, 75(3), pp. 424-440.

Klemperer, P. (1995) Competition when consumers have switching costs: An overview with applications to industrial organization, macroeconomics, and international trade, The Review of Economic Studies, 62(4), pp. 515539.

Kuan, J. W. (2001) Open Source Software as Consumer Integration Into Production, Working paper, SSRN.

Lakhani, K. R. and von Hippel, E. (2003) How open source software works: Free user-to-user assistance, Research Policy, 32(6), pp. 923-943.

Leoncini, R. (2001) Segmentation and increasing returns in the evolutionary dynamics of competing techniques., Metroeconomica, 52(2), pp. 217-237.

Leoncini, R. (2004) Of penguins and innovation, Istituzioni e Sviluppo Economico, 3(2), pp. 57-71.

Lerner, J. and Tirole, J. (2002) Some simple economics of open source, Journal of Industrial Economics, 50(2), pp. 197-234.

Liebowitz, S. J. and Margolis, S. E. (1994) Network externality: An uncommon tragedy, Journal of Economic Perspectives, 8(2), pp. 133-150.

Mansfield, E. (1961) Technical change and the rate of imitation, Econometrica, 29, pp. 741-766.

Medio, A. and Lines, M. (2001) Non linear dynamics. A primer (Cambridge: Cambridge University Press).

Metcalfe, J. (1981) Impulse and diffusion in the process of technological change, Futures, pp. 347-359.

Rossi, M. A. (2006) Decoding the "free/open source(f/oss) software puzzle" a survey of theoretical and empirical contributions, in: J. Bitzer and P. Schroder (Eds.) The Economics of Open Source Development (Elsevier). 
Schmidt, K. M. and Schnitzer, M. (2003) Public Subsidies for Open Source? Some Economic Policy Issues of the Software Market, CEPR Discussion Papers 3793, CEPR.

Shy, O. (2001) The Economics of Network Industries (Cambridge University Press).

Vega-Redondo, F. (2007) Complex Social Networks (Cambridge: Cambridge University Press).

von Hippel, E. and von Krogh, G. (2003) Open source software and the private-collective innovation model: Issues for organization science, Organization Science, 14(2), pp. 209-223.

West, J. (2003) How open is open enough? melding proprietary and open source platform strategies, Research Policy, 32(7), pp. 1259-1285.

Wheeler, D. (2005) Why open source software / free software (oss/fs, floss, or foss)? look at the numbers!, available at http://www.dwheeler.com/oss_fs_why.html .

Witt, U. (1997) "lock-in" vs. "critical masses" - industrial change under network externalities, International Journal of Industrial Organization, 15(6), pp. $753-773$.

\section{Appendix}

\section{A Proof of the asymptotic stability of the equilib- rium}

\section{A.1 Perfect interoperability}

In order to prove the stability of the equilibrium in the case of perfect interoperability analysed in Section 5.1, let us work out the Jacobian of the system at the equilibrium:

$$
\mathbf{J}=\left[\begin{array}{cc}
-\beta_{p}\left(A_{p}^{*}\right) A_{p}^{*} & -\theta_{o p} \beta_{p}\left(A_{p}^{*}\right) A_{p}^{*} \\
-\theta_{p o} \beta_{o}\left(A_{o}^{*}\right) A_{o}^{*} & -\beta_{o}\left(A_{o}^{*}\right) A_{o}^{*}
\end{array}\right]
$$

The discriminant of the associated characteristic equation is:

$$
\begin{aligned}
\Delta & =\left(-\beta_{p}^{*} A_{p}^{*}-\beta_{o}^{*} A_{o}^{*}\right)^{2}-4\left(1-\theta_{p o} \theta_{o p}\right) \beta_{p}^{*} A_{p}^{*} \beta_{o}^{*} A_{o}^{*}= \\
& =\left(\beta_{p}^{*} A_{p}^{*}-\beta_{o}^{*} A_{o}^{*}\right)^{2}+4 \theta_{p o} \theta_{o p} \beta_{p}^{*} A_{p}^{*} \beta_{o}^{*} A_{o}^{*}>0
\end{aligned}
$$

The determinant of $\mathbf{J}$ is positive whereas its trace is negative, therefore both the eigenvalues are real and negative and $\left(A_{p}^{*}, A_{o}^{*}\right)$ is a stable node. 


\section{A.2 Non-perfect interoperability}

In the more general case of non-perfect interoperability (Section 5.2), the Jacobian calculated at the fixed points is:

$$
\mathbf{J}=\left[\begin{array}{cc}
-\beta_{p}\left(A_{p}^{*}\right) A_{p}^{*} & -\left(\frac{1}{\eta_{p o}}+\beta_{p}\left(A_{p}^{*}\right)\right) \theta_{o p} A_{p}^{*} \\
-\left(\frac{1}{\eta_{o p}}+\beta_{o}\left(A_{o}^{*}\right)\right) \theta_{p o} A_{o}^{*} & -\beta_{o}\left(A_{o}^{*}\right) A_{o}^{*}
\end{array}\right]
$$

The discriminant of the associated characteristic equation is thus:

$$
\begin{aligned}
\Delta & =\left(\beta_{p}^{*} A_{p}^{*}+\beta_{o}^{*} A_{o}^{*}\right)^{2}-4\left(\beta_{p}^{*} A_{p}^{*} \beta_{o}^{*} A_{o}^{*}-\left(\frac{1}{\eta_{p o}}+\beta_{p}^{*}\right)\left(\frac{1}{\eta_{o p}}+\beta_{o}^{*}\right) \theta_{p o} \theta_{o p} A_{p}^{*} A_{o}^{*}\right)= \\
& =\left(\beta_{p}^{*} A_{p}^{*}-\beta_{o}^{*} A_{o}^{*}\right)^{2}+4\left(\frac{1}{\eta_{p o}}+\beta_{p}^{*}\right)\left(\frac{1}{\eta_{o p}}+\beta_{o}^{*}\right) \theta_{p o} \theta_{o p} A_{p}^{*} A_{o}^{*}(>0)
\end{aligned}
$$

This discriminant is always positive, whereas the trace of the Jacobian is negative. Thus, the fixed points can be either saddle points or stable nodes depending on the determinant of the Jacobian being positive or negative. This determinant is equal to:

$$
|\mathbf{J}|=\beta_{p}^{*} A_{p}^{*} \beta_{o}^{*} A_{o}^{*}-\left(\frac{1}{\eta_{p o}}+\beta_{p}^{*}\right)\left(\frac{1}{\eta_{o p}}+\beta_{o}^{*}\right) \theta_{p o} \theta_{o p} A_{p}^{*} A_{o}^{*}
$$

and it is positive if and only if:

$$
\theta_{p o} \theta_{o p}\left(1+\frac{1}{\eta_{p o} \beta_{p}^{*}}+\frac{1}{\eta_{o p} \beta_{o}^{*}}+\frac{1}{\eta_{p o} \beta_{p}^{*}} \frac{1}{\eta_{o p} \beta_{o}^{*}}\right)<1
$$

that is, if:

$$
-\frac{\eta_{p o} \beta_{p}^{*}}{\theta_{o p}\left(1+\eta_{p o} \beta_{p}^{*}\right)}<-\theta_{p o}\left(1+\frac{1}{\eta_{o p} \beta_{o}^{*}}\right)
$$

Inequality (17) is satisfied in point $E$ of Figure 5(a). Indeed, in such point we have:

$$
\frac{\mathrm{d} A_{O}^{*}}{\mathrm{~d} A_{p}}<\frac{1}{\frac{\mathrm{d} A_{p}^{*}}{\mathrm{~d} A_{o}}}
$$

Given that, from equation (14) it follows that:

$$
\frac{\mathrm{d} A_{o}^{*}}{\mathrm{~d} A_{p}}>-\frac{\eta_{p o} \beta_{p}^{*}}{\theta_{o p}\left(1+\eta_{p o} \beta_{p}^{*}\right)}
$$

Recalling the symmetry between equation (12) and (13), we have:

$$
-\frac{\eta_{p o} \beta_{p}^{*}}{\theta_{o p}\left(1+\eta_{p o} \beta_{p}^{*}\right)}<\frac{\mathrm{d} A_{o}^{*}}{\mathrm{~d} A_{p}}<\frac{1}{\frac{\mathrm{d} A_{p}^{*}}{\mathrm{~d} A_{o}}}<-\theta_{p o}\left(1+\frac{1}{\eta_{o p} \beta_{o}^{*}}\right)
$$

and the point $E$ is therefore a stable node. 


\section{B Effects of parameter changes on the isocline}

As for $\theta_{o p}$, by the envelope theorem the marginal effect of an increase of it on the maximum of $A_{o}^{*}\left(A_{p}\right)$ - i.e. $A_{o M}^{*}$ - is:

$$
\frac{\partial A_{o M}^{*}}{\partial \theta_{o p}}=-\frac{A_{o M}^{*}}{\theta_{o p}}(<0)
$$

This marginal effect is therefore directly proportional to the initial level of the maximum and it decreases for increasing values of the parameter $\left(\frac{\partial^{2} A_{o M}^{*}}{\partial \theta_{o p}^{2}}>0\right)$.

As for $\eta_{p o}$, its marginal effect is:

$$
\frac{\partial A_{o M}^{*}}{\partial \eta_{p o}}=\frac{A_{o M}^{*}}{\eta_{p o}+\eta_{p o}^{2} \beta_{p}\left(A_{o}^{*-1}\left(A_{o M}^{*}\right)\right)}(>0)
$$

Also this effect is directly proportional to the initial level of the maximum and it decreases for increasing values of the parameter $\left(\frac{\partial^{2} A_{o M}^{*}}{\partial \eta_{p o}^{2}}<0\right)$.

Moreover, a change of $\eta_{p o}$ makes also the value of $A_{p}$ corresponding to $A_{o M}^{*}$ change. In particular, an increase of $\eta_{p o}$ makes $A_{o}^{*-1}\left(A_{o M}^{*}\right)$ decrease.

Indeed, from equation (14) it follows that the FOC are satisfied if the expression in brackets is equal to zero. Working out the total differential of such expression and equating it to zero, after some algebraic manipulation we obtain:

$$
\frac{\mathrm{d} A_{p}}{\mathrm{~d} \eta_{p o}}=-\frac{\beta_{p}\left(A_{p}\right)^{2}}{\beta_{p}^{\prime}\left(A_{p}\right)\left(1+\eta_{p o} \beta_{p}\left(A_{p}\right)+\left(D-A_{p}\right) \frac{\beta_{p}^{\prime}\left(A_{p}\right)}{\beta_{p}\left(A_{p}\right)}\right)-\left(D-A_{p}\right) \beta_{p}^{\prime \prime}\left(A_{p}\right)}
$$

and this expression is always negative.

\section{Stable and unstable equilibria with positive net- work effects across-technologies}

For the sake of simplicity, let us assume a constant propagation coefficient for both PS and OSS and suppose that the increase in the installed base of each software increases the utility of both software for non adopters, so that, besides increasing the probability that each non user will adopt PS, an increase of PS users increases the likelihood that a non user will adopt OSS.

Equations 6 can be modified as follows:

$$
\begin{aligned}
& \dot{A}_{p}=\beta_{p} A_{p}\left(D-A_{p}-\theta_{o p} A_{o}\right)-\frac{\theta_{o p}}{\eta_{p o}} A_{o} A_{p}+\gamma_{p} A_{o} \max \left(D-A_{p}-A_{o}, 0\right) \\
& \dot{A}_{o}=\beta_{o} A_{o}\left(D-A_{o}-\theta_{p o} A_{p}\right)-\frac{\theta_{p o}}{\eta_{o p}} A_{p} A_{o}+\gamma_{o} A_{p} \max \left(D-A_{p}-A_{o}, 0\right)
\end{aligned}
$$


where $\gamma_{p}\left(<\beta_{p}\right)$ and $\gamma_{o}\left(<\beta_{o}\right)$ are non negative parameters measuring the extent of the across-technology positive externalities for PS and OSS respectively. ${ }^{24}$

Given the symmetric formulation, we can analyze the isocline of one technology only. Let us take that of $A_{p}$. It can be written as:

$$
A_{o}=\frac{\eta_{p o} \beta_{p}}{\theta_{o p}\left(1+\eta_{p o} \beta_{p}\right)-\eta_{p o} \gamma_{p} \frac{M}{A_{p}}}\left(D-A_{p}\right)
$$

where $M=\max \left(D-A_{p}-A_{o}, 0\right)$.

It is important to note that this equation is equal to equation 7 , but for the correction term $\eta_{p o} \gamma_{p} M / A_{p}$. However, this term is null when $D \leq$ $A_{p}+A_{o}$. Therefore, when conditions 17 hold, both the isoclines rest above the line $D D$, the correction term does not apply, and the stable equilibrium is the same found in Section 4 (Figure 7(a)). This is because the positive externalities increase the speed of diffusion for both technologies until all the users use at least one software, but the market shares in this case are decided on the co-users.

The case showed in Figure 7(b) is more complex but also in this case the presence of positive externalities across-technologies do not alter the main findings. Indeed, the result is the same of Figure 1(b). In this case however, the isoclines are different. In fact, with reasonable values of the parameters and provided that $\beta>\gamma$, the isoclines are convex curves lying between the $D D$ line and the isoclines for the case of no across-technology externalities (Figure 1(b)). They intersect in the convex hull but the equilibrium is unstable and at the end one of the two software completely displaces the other. ${ }^{25}$

\footnotetext{
${ }^{24}$ These parameters can also be taken as increasing functions of the levels of interoperability. So, for instance: $\gamma_{p}=\gamma_{p}\left(\eta_{p o}\right)$, with $\gamma_{p}^{\prime}\left(\eta_{p o}\right)>0$.

${ }^{25}$ Proofs and examples not included for space constraints and available from the authors at request.
} 


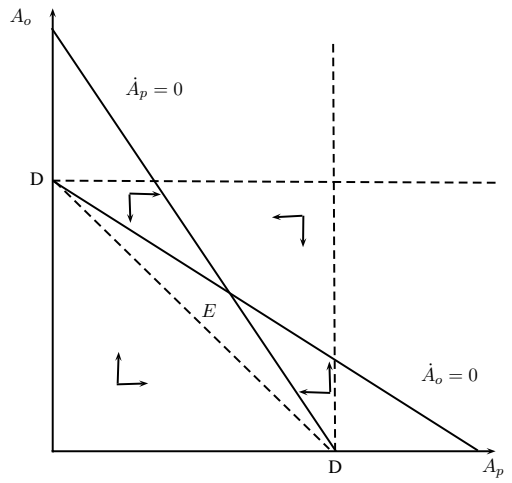

(a) Asymptotically stable equilibrium (b) with technologies' coexistence (high interoperability/low switching costs for both)

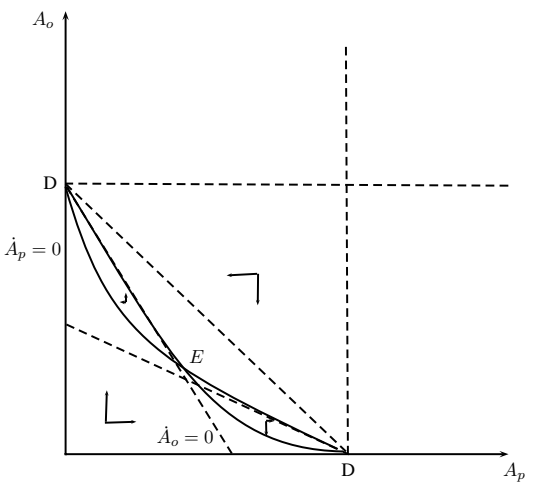

b) Saddle point (low interoperability/high switching costs for both)

Figure 7: Dynamics with constant propagation coefficients and acrosstechnology positive externalities 